\title{
Dynamics Identification of a Monocopter Using Neural Networks
}

\author{
Mostafa Ezabadi, Mohammad Hasan Sabeti, and Afshin Banazadeh
}

\begin{abstract}
In this investigation, a neural network approach is presented for dynamics identification of a single-bladed aerial vehicle or a monocopter. Implementation of neural networks let us do the non-parametric identification process regardless of the system dynamics. Here, we have initially designed a feedforward network and found that this approach is insufficient for the mentioned purpose. Therefore, a novel network with NARX structure with one hidden layer, tansig activation function and 15 neurons is designed and excellent results are obtained due to consideration of past outputs in the training process.
\end{abstract}

Index Terms-Monocopter, dynamics identification, feedforward neural network, NARX.

\section{INTRODUCTION}

System identification for complex dynamics by means of conventional methods is highly time-consuming and requires practical flight tests. Significant advancements in the field of small air vehicles have caused a widespread demand. Single-bladed aerial vehicle or monocopter is a type aerial vehicles which has drawn many attentions in recent years. This vehicle is able to carry out all flight phases like a conventional helicopter including hover, vertical takeoff and landing and forward flight, with simpler configuration compared to helicopters (Fig. 1). It is capable of producing high aerodynamic efficiency due to its dynamics [1], [2] .

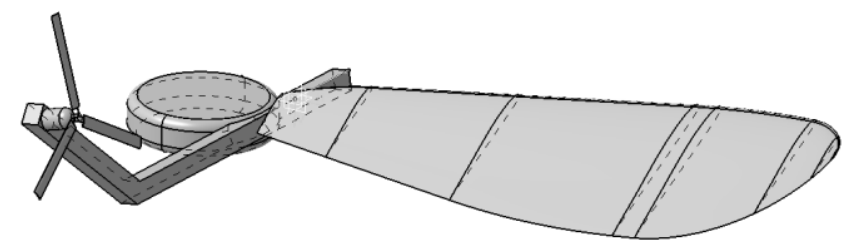

Fig 1. Schematic of the monocopter.

The idea of this vehicle was first conceived by falling of a maple seed. Maple seed structure consists of a tiny wing with a concentrated mass attached to its root. This structure causes a spiral motion when it falls down from the tree. The first artificial vehicle was flown in 1952 by Charles W. McCutchen [3]. In 1991, Rosen and Seter, Developed an experimental-based model of autorotation for maple seed [4]. These two, in 1992, investigated stability of maple seed in autorotation in comparison to numerical models and found the stability derivatives of maple seed [5]. In 2010, Evan Ulrich, presented a linear model using frequency domain identification for hovering flight and designed a controller for it [6], [7]. In 2015, Banazadeh et al presented modeling and simulation of a single-bladed aerial vehicle using

Manuscript received on January 16, 2017; revised May 1, 2017.

The authors are with the Department of Aerospace Engineering, Sharif University of Technology, Tehran, Iran (e-mail: kebriaee@ sharif.ir). blade-element momentum theory [8]. In 2015, Matič et al gave a mathematical model based on unsteady blade-element momentum theory [1].

In [8], Banazadeh and et al developed seven degrees of freedom multibody model of a monocopter air vehicle based on the Newton-Euler approach along with nonlinear simulation in different flight phases. For modeling of aerodynamic forces and moments, they used blade element momentum theory. In suggested configuration for monocopter, the sole control surface is modeled like a conventional flap on a wing. Free flight simulation is performed in MATLAB Simulink environment to evaluate the behavior of the system and to demonstrate the effectiveness and applicability of the proposed model. Simulation results show linear and angular velocities are consistent with the physics and mathematical foundation s. Static stability of the vehicle is evident in free flight by careful choice of initial conditions.

In this paper, it is going to determine the simulated model using N.N. in order to design a proper controller for it.

\section{NetWork STRUCTURE}

For the purpose of identification, two kinds of networks are used: feedforward and NARX network. We first used feedforward network structure because this kind of structure popular for curve fitting problems [9]. On the other hand, NARX network structure, as a dynamic structure is more effective for the identification of single-bladed aerial vehicle. This network gives the best output in comparison with other dynamic networks [9]. General structures for both networks are given below:

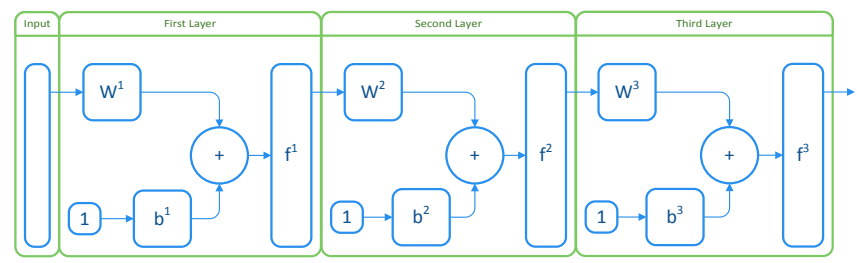

Fig. 2. Schematicof the feedforward network.

As seen in Fig. 4 and 5, sensitivity analysis is also performed on the following parameters. In feedforward network, number of hidden layers and number of neurons are studied. Activation function in each layer was "logsig" and Levenburg-Maquardt learning rule was considered for training algorithm. LM algorithm has been implemented due to its capability to minimize the error by correcting weights and biases through back-propagating of the errors from the last layer to the first. After the training process, step and ramp inputs are implemented for the purpose of validation. 


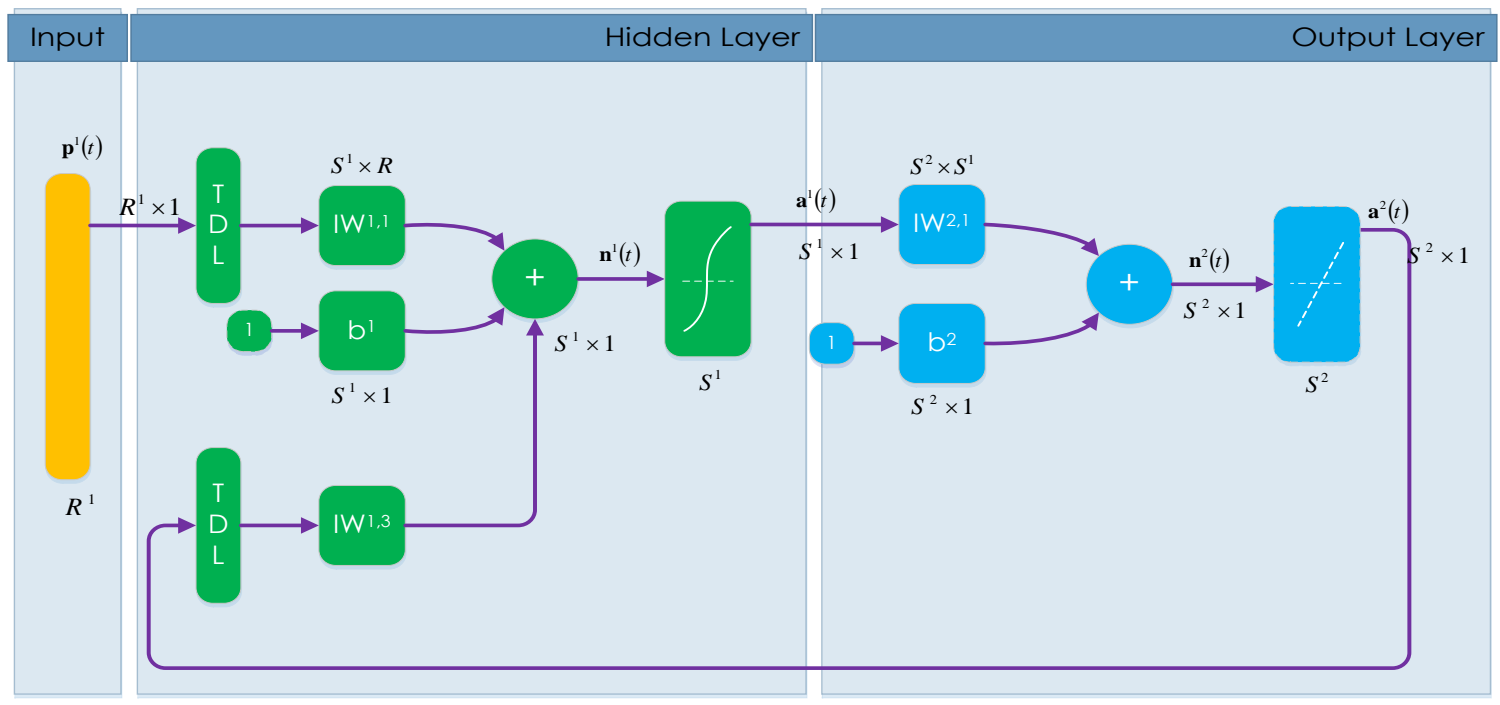

Fig. 3. Schematic of the NARX network.

\section{RESULTS AND DISCUSSION}

\section{A. 5 Neurons in the Hidden Layer}

For initial investigation, we have considered 5 neurons in the hidden layer of feedforward network. The simulation results are given in Fig. 6. As shown in this Fig. 5 neurons in hidden layer are not sufficient so the network is not capable of capturing the vehicle's complex dynamics.

\section{B. 15 Neurons in the Hidden Layer}

For next investigation, we have considered 15 neurons in the hidden layer. The simulation results are given in Fig. 7. The results are not still perfect, although increasing the number of neurons makes better results.

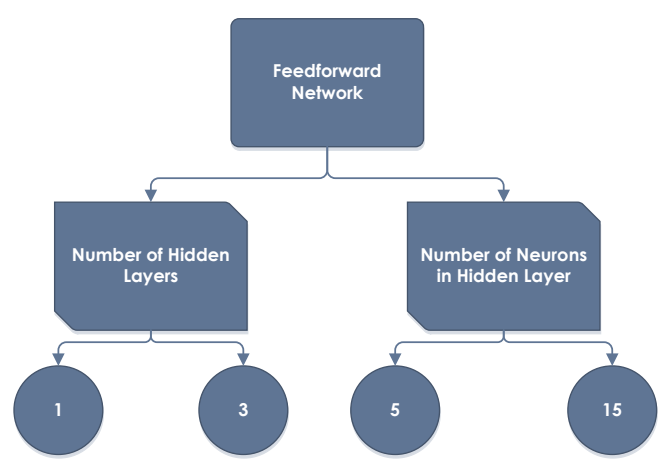

Fig. 4. Feedforward network parameters.

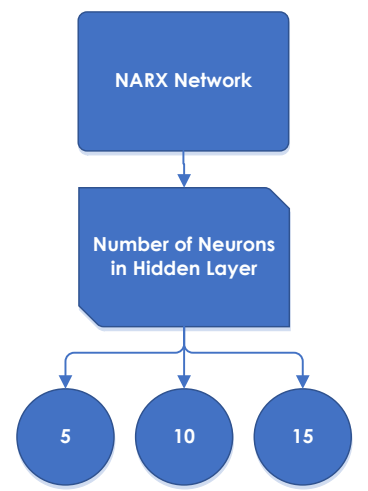

Fig. 5. NARX network parameters.
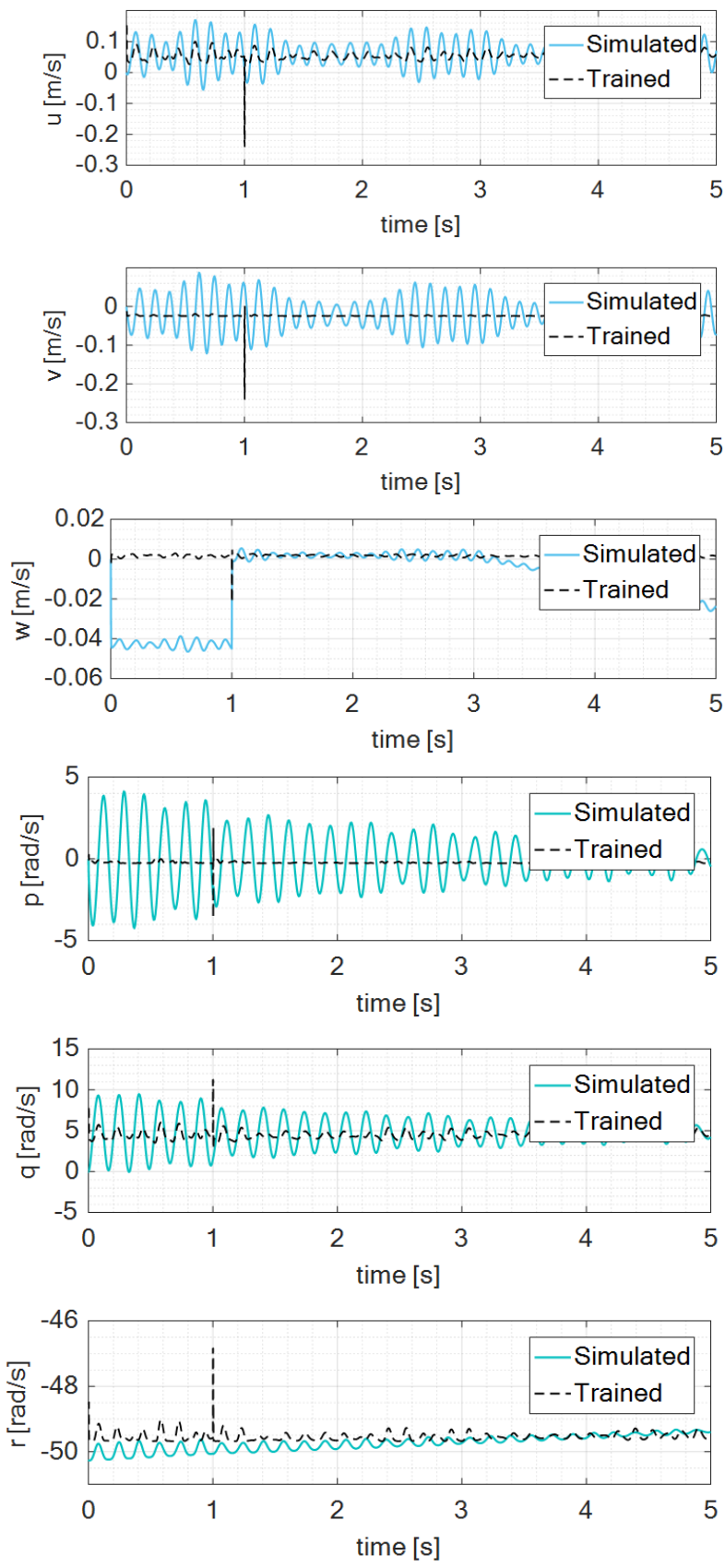

Fig. 6. Linear and angular velocities for 5 neurons in the hidden layer. 

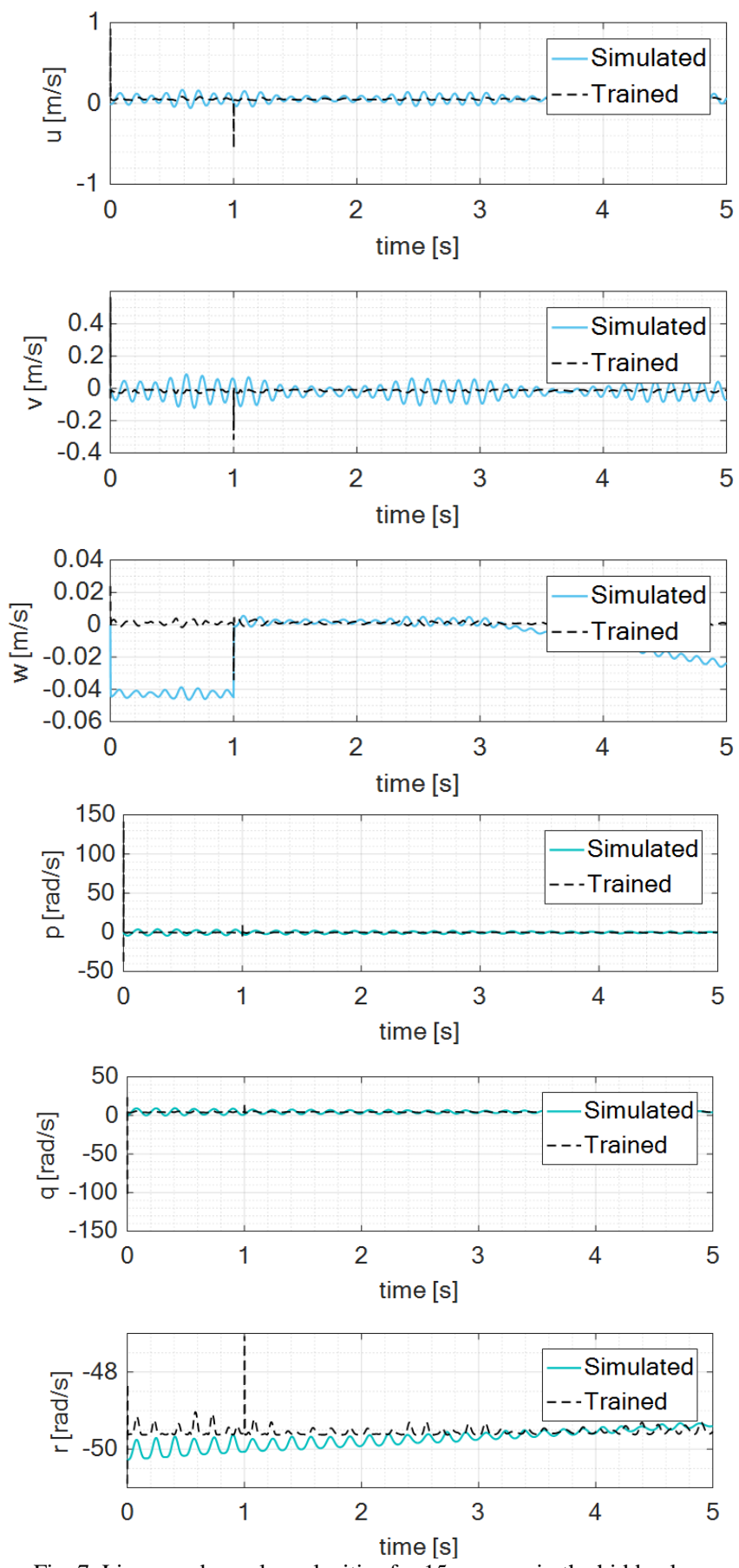

Fig. 7. Linear and angular velocities for 15 neurons in the hidden layer.

\section{Effect of Hidden Layers}

Vehicle's dynamics is very complex. So, one hidden layer is not likely to be sufficient for the identification process. Here, we have considered three hidden layers and 15 neurons in each layer in the feedforward network. As the results in Fig. 8 shows, increasing the number of hidden layers is not still adequate for dynamics identification of this kind of air vehicle Whatever the number of neurons and hidden layers are changed this kind of structure is not capable to identification would urge a dynamic network.

\section{NARX Network}

Feedforward network is a static one, which uses instant inputs and outputs for training process. This structure was not able to identify given dynamics, so a dynamic structure is proposed here for this purpose. This structure utilizes past inputs and outputs in addition to the instantaneous inputs and outputs. We have chosen NARX network that is very popular for the identification purpose.
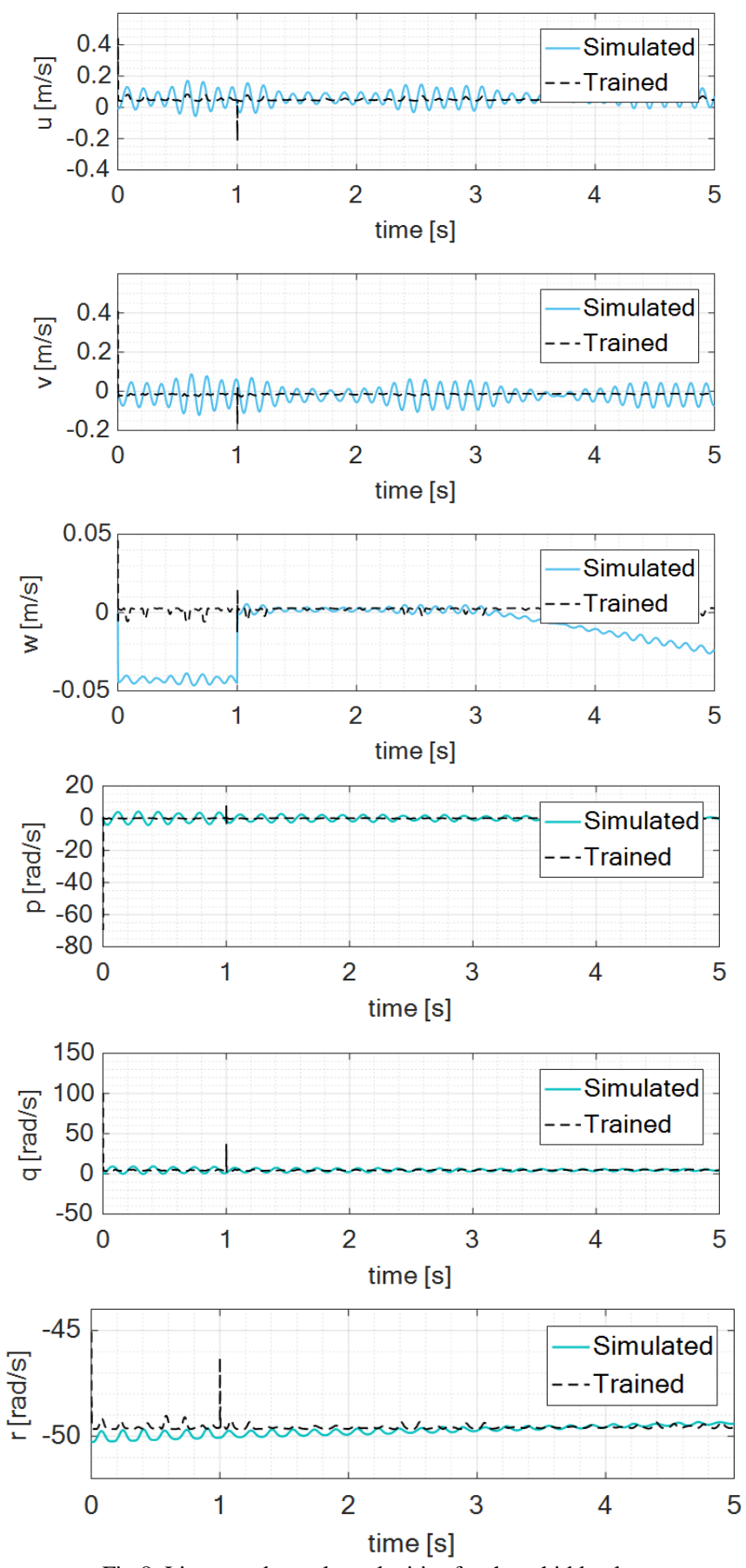

Fig 8. Linear and angular velocities for three hidden layers.

\section{E. Effect of Number of Neurons in Hidden Layer}

For initial investigation, we have considered 5 neurons in the NARX hidden layer. The simulation results are presented in Fig. 9. The results show that this kind of network can predict more accurate results in comparison with the feedforward network. However, the number of neurons could be increased regarding the vertical velocity estimation, w. Increasing the number of neurons to 15 gives the capability of producing more accurate outputs, which leads to perfect identification as presented in Fig. 10. 

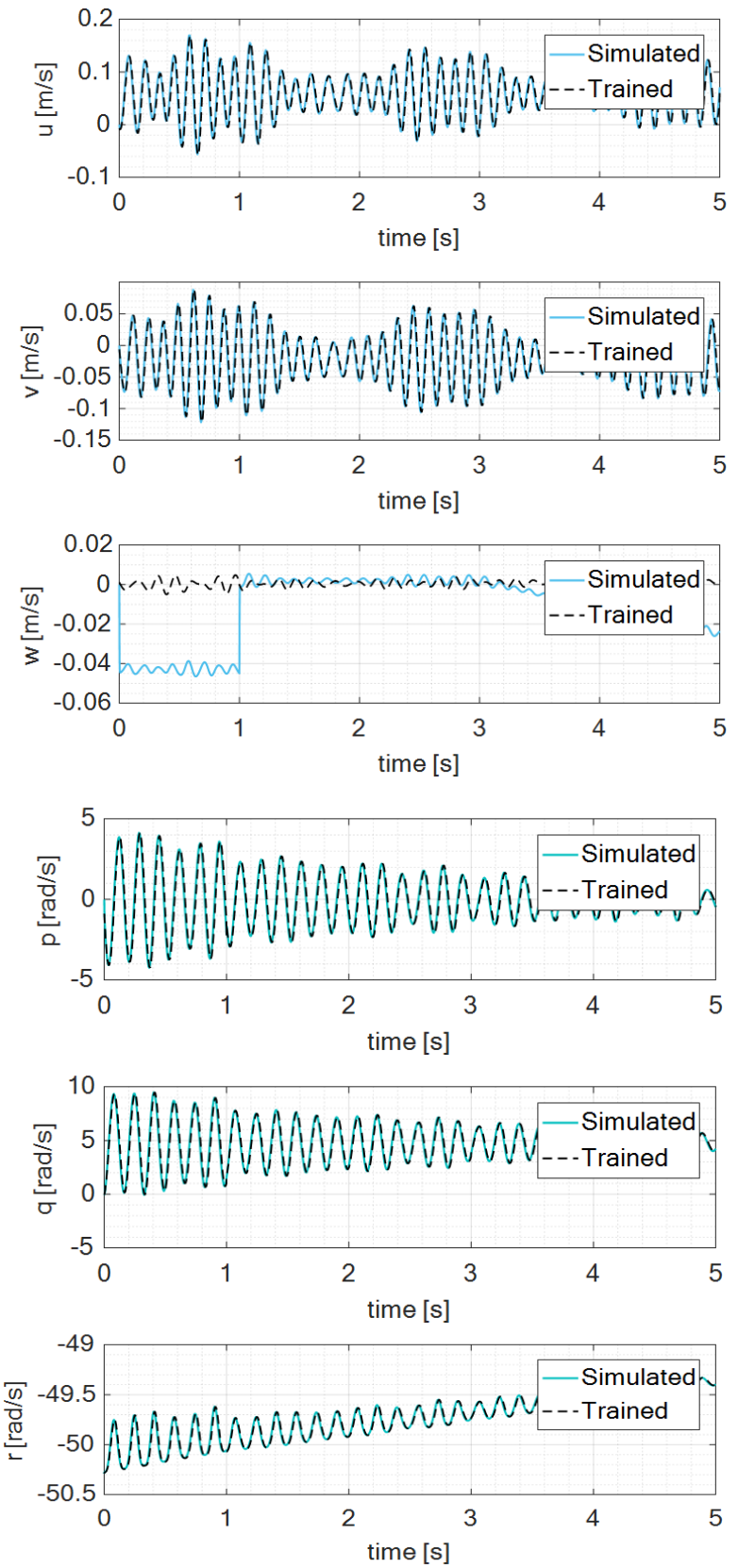

Fig 9. Linear and angular velocities for 5 neurons in the hidden layer.
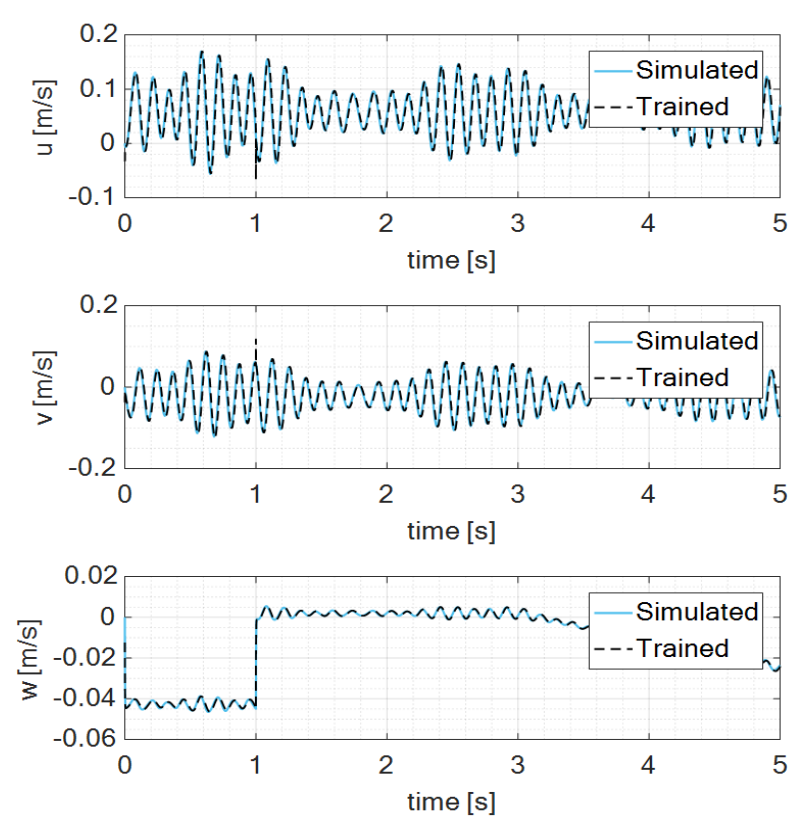
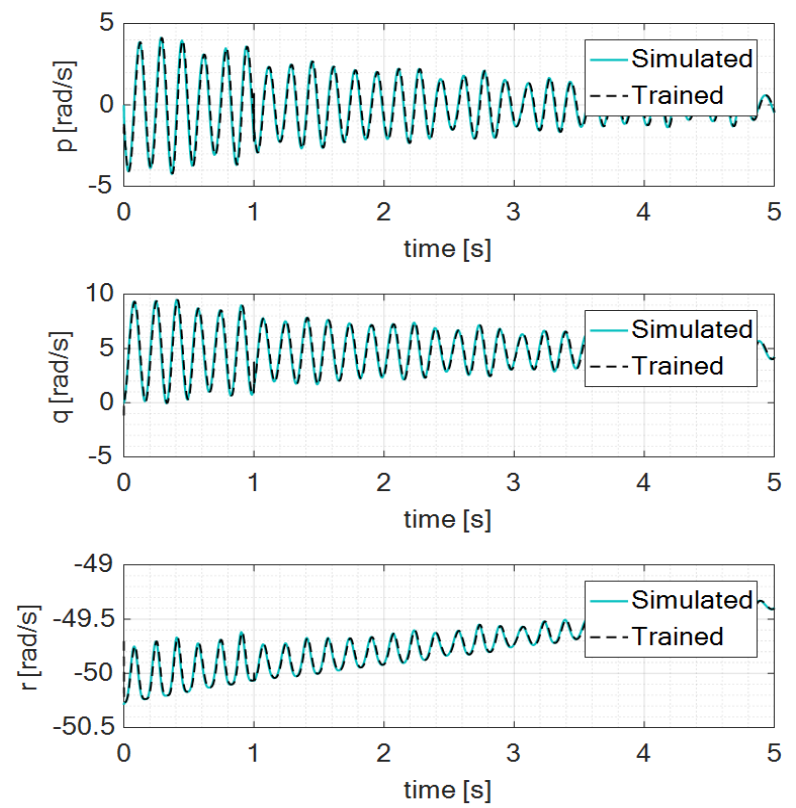

Fig. 10. Linear and angular velocities for 5 neurons in the hidden layer.
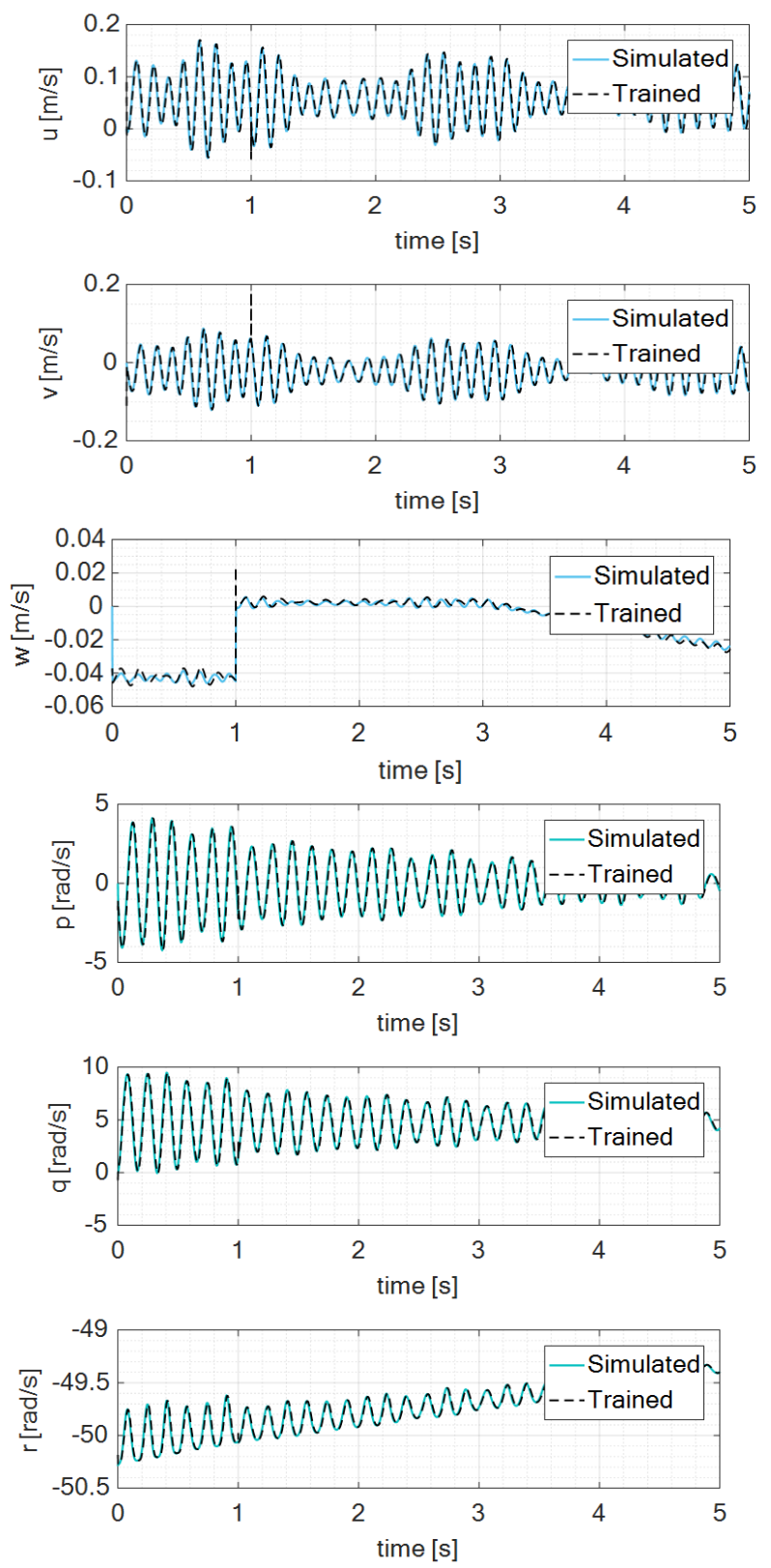

Fig. 11. Linear and angular velocities for 15 neurons in the hidden layer. 


\section{CONCLUDING REMARKS}

Identification of single-bladed aerial vehicle has not yet been performed. In this paper, dynamic identification of a monocopter with the help of neural networks was presented. Feedforward network with different structures was designed that was not able to correctly identify the vehicle dynamics. A NARX network is then designed with one hidden layer, 15

\section{REFERENCES}

[1] G. Matič, M. Topič, and M. Jankovec, "Mathematical model of a monocopter based on Unsteady blade-element momentum theory," Journal of Aircraft, vol. 52, pp. 1905-1913, 2015.

[2] B. Obradovic, G. Ho, R. Barto, K. Fregene, and D. Sharp, "A multi-scale simulation methodology for the samarai monocopter micro UAV," in Proc. AIAA Modeling and Simulation Technologies Conference, vol. 4, pp. 1-12, 2012.

[3] E. R. Ulrich, I. Faruque, J. Grauer, D. J. Pines, J. S. Humbert, and J. E. Hubbard, "Control model for robotic samara: Dynamics about a coordinated helical turn," Journal of Guidance, Control, and Dynamics, vol. 33, pp. 4-9, 2010

[4] A. Rosen and D. Seter, "Vertical autorotation of a single-winged samara," Journal of Applied Mechanics, vol. 58, p. 1064, 1991.

[5] D. Seter and A. Rosen, "Stability of the vertical autorotation of a single-winged samara," Journal of Applied Mechanics, vol. 59, p. 1000, 1992.

[6] E. Ulrich, J. Grauer, D. Pines, J. H. Jr, and J. S. Humbert, "Identification of a robotic samara aerodynamic / multi-body dynamic model," AIAA Atmospheric Flight Mechanics Conference, 2010.

[7] E. R. Ulrich, J. S. Humbert, and D. J. Pines, "Pitch and heave control of robotic samara micro air vehicles," Journal of Aircraft, vol. 47, pp. 1290-1299, 2010.
[8] M. E. Dormiyani, A. Banazadeh, and F. Saghafi, "Multibody modeling and simulation of monocopter micro air vehicle," Applied Mechanics and Materials, vol. 772, p. 401, 2015.

[9] H. B. Demuth, M. H. Beale, O. De Jess, and M. T. Hagan, Neural Network Design, Martin Hagan, 2014

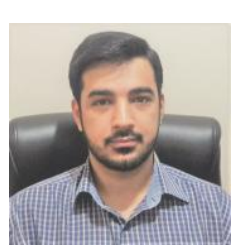

Mostafa Ezabadi was born in Tehran, Iran in 1993 He obtained his bachelor degree in aerospace engineering from Sharif University of Technology in 2015 and is currently the M.Sc. student in flight dynamics and control. His main areas of research interest are neural networks, modeling, and control.

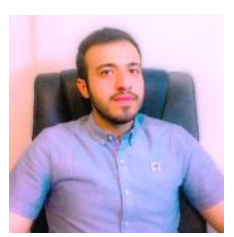

Mohammad Hasan Sabeti was born in Hamadan, Iran in 1993. He received his B.Sc. in aerospace engineering from Sharif University of Technology in 2015 and is currently the M.Sc. student in flight dynamics and control. His research interests include airplane design, neural networks, and identification.

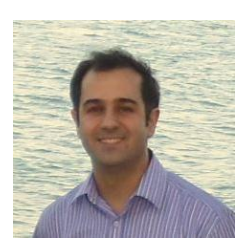

Afshin Banazadeh is an associate professor in the Faculty of Aerospace Engineering at Sharif University of Technology. He received his Ph.D in 2008 from Aerospace Engineering Department at SUT, where he also received his M.Sc. in flight dynamics and B.Sc. in mechanical engineering. His current research interests span system identification, aircraft design and flight test engineering. Besides, he consults for industry on matters dealing with modeling, simulation and control, as well as technology demonstration schemes. 